\section{Paracetamol bringt bei Rückenschmerzen nichts}

\author{
Die Ergebnisse einer australischen Metaanalyse bestätigen, dass Para- \\ cetamol bei Rückenschmerz die Beschwerden innerhalb von 3 Monaten \\ nicht besser lindert als Placebo. Bei Patienten mit Hüft- oder Kniearthrose \\ ist die Schmerzlinderung nicht klinisch relevant.
}

$\mathrm{N}$ och immer wird in vielen Leitlinien weltweit bei Rücken- und Nackenschmerzen sowie Hüft- und Kniearthrose Paracetamol für die Ersttherapie empfohlen. Für ihre aktuelle systematische Übersicht und Metaanalyse haben Wissenschaftler um Gustavo C. Machado, Sydney, Australien, randomisierte, placebokontrollierte Studien ausgewählt. Primäre Endpunkte waren Schmerz, Einschränkungen der Beweglichkeit und Lebensqualität. 13 aus 116 Studien ließen sich für die Analyse nutzen.

In 10 Studien mit 3.541 Patienten wurde die Wirksamkeit von Paracetamol bei Hüft- und Kniearthrose geprüft, in 3 Studien mit 1.825 Patienten bei Rückenschmerzen. Auf der Suche nach geeigneten Studien zu Nackenschmerzen wurden die Wissenschaftler nicht fündig. In den meisten Studien wurde das Medikament als Tablette oder Kapsel verabreicht, nur in einer Studie bei Rückenschmerz intravenös (1.000 mg einmalig). Die Dosis lag bei $3.000 \mathrm{mg}$ pro Tag (oral) bzw. zwischen 3.900 und $4.000 \mathrm{mg} / \mathrm{Tag}$. Das Follow-up lag höchstens bei $6 \mathrm{Mo}$ naten. Informationen über Langzeiteffekte, also über 1 Jahr hinaus, konnten aus den Studien nicht gewonnen werden.

Die Qualität der Evidenz wurde mithilfe des GRADE („Grading of Recom-

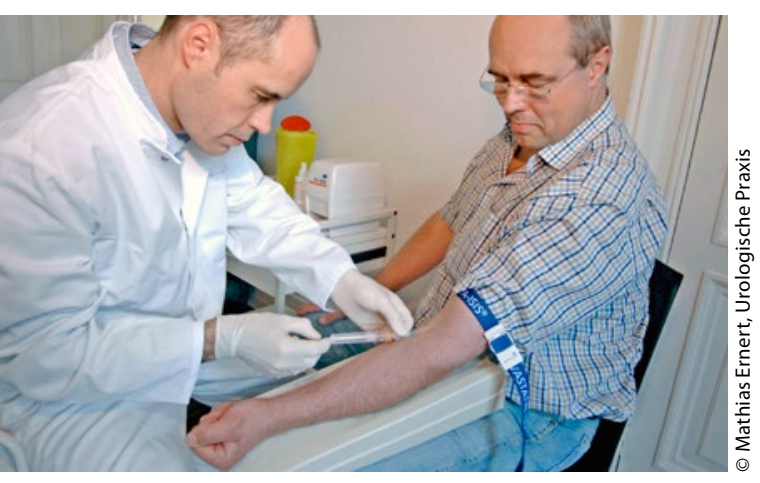

Die Chance für verschlechterte Leberfunktionswerte ist unter Paracetamol 4-mal höher als unter Placebo. mendations Assessment, Development and Evaluation")-Systems in 4 Kategorien eingestuft, von "high quality“ bis „very low quality“. Für die Beurteilung der Schmerzstärke und Beweglichkeit wurde auf der Grundlage der visuellen Analogskala und des Scores WOMAC („Western Ontario and McMaster Universities Osteoarthritis Index") eine Punkteskala von 0 (kein Schmerz bzw. keine Einschränkung der Beweglichkeit) bis 100 (stärkster Schmerz bzw. vollkommen eingeschränkt) verwendet. $\mathrm{Ne}$ gative Werte bei der Differenz entsprechen einem besseren Abschneiden von Paracetamol.

Aufgrund der statistischen Auswertung kommen Machado und seine Kollegen zu dem Schluss, dass Paracetamol zur Schmerzreduktion im Rücken nicht geeignet ist. Im Vergleich zu Placebo betrug über einen Zeitraum bis zu 3 Monaten die Differenz der Schmerzintensität nur -0,5 Punkte (95\%-Konfidenzintervall [95\%-KI] zwischen -2,9 und 1,9). Beim Parameter Beweglichkeit lag die Differenz bei 0,4 Punkten (95\%-KI zwischen -1,7 und 2,5). Auch die Lebensqualität war in den Verumgruppen nicht besser als in den Vergleichsgruppen. Die Evidenzqualität war jedes Mal hoch.

Etwas anders lagen die Ergebnisse bei der Auswertung der Studien zur Arthrose. Paracetamol linderte die Schmerzen zwar signifikant stärker als Placebo (-3,7 Punkte; 95\%-KI zwischen -5,5 und $-1,9)$ sowie die Beweglichkeit $(-2,9$ Punkte; 95\%-KI zwischen -4,9 und $-0,9)$ - jeweils innerhalb von 3 Monaten. Doch sei dieser Effekt klinisch nicht relevant, so die Forscher, was für alle Werte unter 4 Punkten auf der Skala zwischen 0 und 100 gelte.

Außerdem war die Wahrscheinlichkeit für anormale Ergebnisse von Leberfunktionstests in 3 Studien mit fast 1.300 Patienten während der Paracetamol-Therapie erhöht: Die Wahrschein- lichkeit, dass die Leberfunktionswerte Aspartattransaminase (AST)/Alanintransaminase (ALN) über dem 1,5-Fachen der oberen Normgrenze liegen, war bei Patienten mit Paracetamol-Behandlung im Vergleich zur Placebogruppe fast 4-mal höher (Risikoverhältnis: 3,8; $95 \%$-KI zwischen 1,9 und 7,4).

Fazit: Machado und seine Kollegen schlagen vor, aufgrund dieser Studienergebnisse die Empfehlungen für die Anwendung von Paracetamol in vielen Leitlinien zu überdenken. In der bis November 2015 gültigen „Nationalen VersorgungsLeitline Kreuzschmerz“ etwa heißt es, dass aufgrund geringer Evidenz und möglicher Nebenwirkungen Paracetamol nicht als Mittel der ersten Wahl empfohlen werden kann. Und: „Es kann für einen Behandlungsversuch angewendet werden, wobei eine Besserung der Schmerzen innerhalb einer Woche eintreten sollte.“

Peter Leiner

Machado GC et al. Efficacy and safety of paracetamol for spinal pain and osteoarthritis: systematic review and meta-analysis of randomised placebo controlled trials. BMJ. 2015;350:h1225.

\section{Kommentar von Prof. Diener:}

Dies ist innerhalb von $1 \mathrm{Jahr}$ die dritte große Metaanalyse, die belegt, dass Paracetamol in der Behandlung von Rückenschmerzen nicht besser wirksam ist als Placebo. Das Ergebnis wird unterstützt durch eine weitere, erst kürzlich durchgeführte große randomisierte Studie in Australien. Daher wäre es jetzt Zeit, Paracetamol aus den Therapieempfehlungen zum Rückenschmerz zu verbannen. Dies ist auch notwendig, da es bei den relativ hohen Dosen von Paracetamol immer wieder zu Leberschäden kommt. Der geringe therapeutische Effekt bei Knie- und Hüftgelenksarthrose rechtfertigt ebenfalls nicht den Einsatz von Paracetamol. Für beide Krankheitsbilder sollten daher nicht steroidale Antirheumatika und gegebenenfalls retardierte Opioide eingesetzt werden.

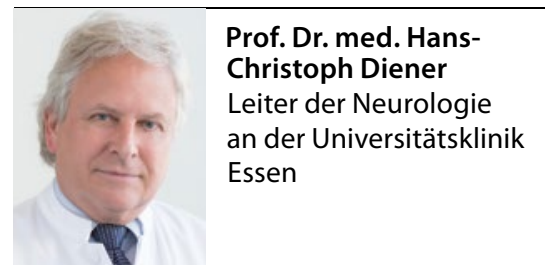

